\title{
TITLE:
}

\section{Which classical correspondence is valid in intuitionistic modal logic?}

$\operatorname{AUTHOR}(\mathrm{S})$ :

Kojima, Kensuke

\section{CITATION:}

Kojima, Kensuke. Which classical correspondence is valid in

intuitionistic modal logic?. Logic Journal of IGPL 2012, 20(1): 331-348

\section{ISSUE DATE:}

2012-12-01

URL:

http://hdl.handle.net/2433/166077

\section{RIGHT:}

(c) The Author 2011. Published by Oxford University Press.; この論文は出 版社版でありません。引用の際には出版社版をご確認ご利用ください 。; This is not the published version. Please cite only the published version. 


\title{
Which Classical Correspondence is Valid in Intuitionistic Modal Logic?
}

\author{
Kensuke Kojima \\ Graduate School of Informatics, Kyoto University
}

\begin{abstract}
Modal logics reason about properties of relational structures, and such properties are often characterized by axioms of modal logics. This connection between properties of relational structures and axioms of modal logics are called correspondence, and has been investigated well in the classical setting.

The problem we consider is an intuitionistic version of this correspondence. In particular, this paper considers which part of the correspondence results known in classical setting is true for intuitionistic one.

We first define the notion of robustness of axioms so that an axiom is robust if and only if its corresponding properties in classical and intuitionistic semantics are the same. Next we give a syntactically defined class of axioms, and prove that all axioms in this class are robust. This result is an analogue of the classical result by Sahlqvist, and its proof is partly based on a known proof of his theorem.
\end{abstract}

\section{Introduction}

\subsection{Background and Main Result}

Modal logics describe various properties of relational structures such as reflexivity, transitivity, seriality, etc. These properties are often characterized by axioms of modal logics. For example, reflexivity is characterized by axiom $\mathbf{T}$ $(\square p \rightarrow p)$ in the sense that a structure is reflexive if and only if it validates this axiom. Similarly, transitivity and seriality are characterized by axioms 4 $(\square p \rightarrow \square \square p)$ and $\mathbf{D}(\square p \rightarrow \diamond p)$, respectively.

This relationship between properties and axioms is known as correspondence, and has been investigated in the area of (classical) modal logic [5]. Correspondence theory studies how modal axioms and properties of relational structures are related to each other. It describes an intuitive meaning of a modal axiom in terms of relational structures, and as a result we can know what kind of structure is implied by the modal axiom under consideration. Also, when we have some property of relational structure we are interested in, from correspondence theory we can know which axiom characterizes that property. 
When reasoning about modal logics, we often find it helpful to move back and forth between syntactic and semantic entities (modal axioms and relational properties). An intuitionistic version of the correspondence theory will be useful in understanding and developing intuitionistic modal logics. Unfortunately, however, it seems that an intuitionistic version of the correspondence theory has not been extensively studied before.

This paper considers correspondence in intuitionistic setting. In particular, we consider which part of the existing classical correspondence results is also true for intuitionistic one. More precisely, the problem we consider is as follows. First, consider an axiom $X$ we are interested in. Then, on the one hand, we have a correspondent $\varphi$ of $X$ in classical modal logic. On the other hand, we have another correspondent $\psi$, in intuitionistic modal logic. This paper studies when the two properties $\varphi$ and $\psi$ are the same.

For example, correspondence results for $\mathbf{T}, \mathbf{4}$ and $\mathbf{D}$ mentioned above apply to intuitionistic modal logic. ${ }^{1}$ However, for axiom $\mathbf{5}(\diamond p \rightarrow \square \diamond p)$, it is not the case. This axiom classically corresponds to Euclidean property, ${ }^{2}$ but intuitionistically it does not; in intuitionistic modal logic, there exists a non-Euclidean frame satisfying $\mathbf{5}$ (such an example will appear in Section 3).

We have two technical contributions in this paper:

1. We introduce the notion of "robustness." This notion is defined in a fairly simple way, but still captures the "sameness" of the two correspondents (one for classical, and the other for intuitionistic).

2. We give a sufficient condition for robustness. We define a class of axioms in a syntactic way, and prove that all of its members are robust.

Behind this result there is a classical result known as Sahlqvist's theorem. This theorem gives a sufficient condition for axioms to correspond to a first-order property [15]. The idea on which our result is based is similar to the idea found in this classical result.

\subsection{Organization of This Paper}

Section 2 gives the foundation of our work. First we take a look at some previous approaches to Kripke semantics for intuitionistic modal logics, and then introduce a semantics by Wolter and Zakharyaschev [21]. After that, in the first part of Section 3, we discuss some examples of classical correspondence results in intuitionistic semantics introduced in Section 2. The second part introduces the notion of "robustness," with justification of its definition. In Section 4, we state and prove the main theorem, a sufficient condition for the robustness. Thanks to simplicity of the definition of robustness, combined with algebraic representation of Kripke semantics, the proof is fairly simple. As an example of

\footnotetext{
${ }^{1}$ Actually there are several ways to define Kripke semantics for intuitionistic modal logic, and corresponding properties depend on the choice of semantics. The results here is the case of IM-frames, defined in Section 2.

${ }^{2} \mathrm{~A}$ binary relation $R$ is said to be Euclidean if it satisfies: if $x R y$ and $x R z$, then $y R z$.
} 
possible application, in Section 5 we consider axioms arising in the application to security. We show some of them are robust, and using this fact we compare strength of these axioms. Section 6 gives examples of robust and non-robust axioms. Section 7 summarizes this paper, and makes some remarks, including related work.

\subsection{Notational Conventions}

Through this paper, we use the following notation.

- A binary operator $(\cdot ; \cdot)$ denotes the composition of binary relations: if $R$ and $S$ are binary relations, $x(R ; S) y$ if and only if $\exists z .(x R z) \wedge(z S y)$.

- For a binary relation $R$ on a set $X$ and an element $x \in X$, its image $\{y \mid x R y\}$ is denoted by $R[x]$.

- For some notions introduced below, we have both classical and intuitionistic versions. In such a case, we use annotations $c l$ and int to distinguish these versions.

The symbol $*$ is also used instead of $c l$ or $i n t$. If $*$ appears in an equality, equivalence or other statement, it applies to both classical and intuitionistic versions.

\section{Preliminaries}

For intuitionistic modal logics, there are more than one way to define semantics, even if we only consider Kripke-style semantics. These variants are often discussed in the literature.

This section gives a brief overview of the existing approaches, and introduces the semantics using IM-frames, which is first considered by Wolter and Zakharyaschev [21] and our work is based on.

\subsection{Syntax of Formulas}

First of all, we fix the syntax of formulas used below. Formulas we are going to consider are generated from a fixed set of atoms, by propositional connectives $\wedge, \vee, \rightarrow$, necessity operator $\square$, and possibility operator $\diamond$. We assume that the set of atoms contains constants $\perp$ and $\top$, and as usual we define negation $\neg A$ by $A \rightarrow \perp$.

Below $p$ and $q$ range over the set of atoms other than $\perp$ and $\top$, and $A$ and $B$ range over formulas.

\subsection{Existing Kripke Semantics}

As Kripke-style semantics for intuitionistic modal logics, it seems that there are the following two main approaches in the literature: 
1. Interpret intuitionistic modal logic in a model of intuitionistic first-order logic, by correlating modal operators and first-order quantifiers.

2. Start from Kripke semantics in the classical sense, and augment it with some structure which represents intuitionistic counterpart.

The first one is an intuitionistic analogue of the well-known relationship between classical modal logic and classical first-order logic. Accordingly, $\square$ and $\diamond$ are interpreted in the same way as intuitionistic $\forall$ and $\exists$. Kripke semantics based on this idea is studied by Ewald [8] and Simpson [17], for example.

In the second approach, there are some possible choices on an additional structure to be added. Perhaps the most common approach is to add another accessibility relation $\leq$ (taken from the standard Kripke semantics for intuitionistic logic) to Kripke frames [19, 3, 14, 17, 18, 7, 21, 20]. As a result, Kripke frames given in this way have two accessibility relations, and are called birelational frames. When doing this, there are some choices in how the intuitionistic accessibility $\leq$ should interact with the modal one $R$, and there are subtle differences among them [3].

Instead of adding intuitionistic accessibility relation, changing the set of truth-values is another way previously considered to introduce intuitionistic behavior. Namely, we can take truth-values from some Heyting-algebra instead of $\{0,1\}$. This approach is studied by Ono as "modal-type Kripke models" [13]. Also, other variants which use neighborhood frames instead of Kripke frames are studied by Sotirov [18] and Wijesekera [19].

\subsection{Kripke IM-frame and Semantics}

In this paper, we consider Kripke semantics using Kripke IM-frame defined by Wolter and Zakharyaschev [21]. They originally studied not only Kripke frames, but also general frames. They defined IM-frames as general frames equipped with an ordering (which expresses intuitionistic counterpart), but in this paper we simply say IM-frame to mean Kripke IM-frame in their terminology. Since we do not consider general frames here, no confusion occurs.

Definition 1. 1. A (Kripke) IM-frame is a triple $\langle W, \leq, R\rangle$ where $W$ is a non-empty set, $\leq$ is a partial order on $W$, and $R$ is a binary relation on $W$ satisfying $(\leq ; R ; \leq)=R$. We say that $X \subseteq W$ is upward-closed if $w \leq w^{\prime}$ and $w \in X$ implies $w^{\prime} \in X$ for all $w, w^{\prime} \in W$.

2. A valuation $V$ on an IM-frame $\mathcal{F}=\langle W, \leq, R\rangle$ is a map from the set of atoms to the powerset of $W$ such that $V(\perp)=\emptyset$ and $V(\top)=W$. We say that a valuation $V$ is intuitionistic if $V(p)$ is upward-closed for all atoms $p$.

Below we use $\mathcal{F}$ to denote an IM-frame. $W, \leq, R$ and $V$ are also used in the same meaning as the definition above.

Next we define classical and intuitionistic interpretations of modal formulas. We use $\mathcal{F}, V, w \Vdash^{c l} A$ to denote that $A$ is classically true at world $w$ in the frame 
$\mathcal{F}$ under the valuation $V$. When $\mathcal{F}$ and $V$ are clear from context, we simply write $w \Vdash^{c l} A$. For the intuitionistic interpretation, we use $\Vdash^{\text {int }}$ instead of $\Vdash^{c l}$.

Usually the classical interpretation is defined on a pair $\langle W, R\rangle$ (i.e. a Kripke frame in the classical sense) rather than a triple, but ignoring the intuitionistic part $\leq$ of IM-frame we regard IM-frames as Kripke frames. So, by abuse of notation, we use the same $\mathcal{F}$ for both classical and intuitionistic interpretations.

Definition 2. For an IM-frame $\mathcal{F}$, a valuation $V$ on $\mathcal{F}$, a possible world $w$ in $\mathcal{F}$, and a formula $A$, we define relations $\mathbb{}^{c l}$ and $\Vdash^{\text {int }}$ by the following clauses (we omitted $\mathcal{F}$ and $V$ because they are fixed through the definition). For $\Vdash^{\text {int }}$, we assume that $V$ is an intuitionistic valuation.

- $w \Vdash^{*} p \Longleftrightarrow w \in V(p)$

- $w \Vdash^{*} A \wedge B \Longleftrightarrow w \Vdash^{*} A$ and $w \Vdash^{*} B$

- $w \Vdash^{*} A \vee B \Longleftrightarrow w \Vdash^{*} A$ or $w \Vdash^{*} B$

- $w \Vdash^{*} \square A \Longleftrightarrow$ for all $w^{\prime}$, if $w R w^{\prime}$ then $w^{\prime} \Vdash^{*} A$

- $w \Vdash^{c l} A \rightarrow B \Longleftrightarrow$ if $w \Vdash^{c l} A$ then $w \Vdash^{c l} B$

- $w \Vdash^{i n t} A \rightarrow B \Longleftrightarrow$ for all $w^{\prime}$, if $w \leq w^{\prime}$ and $w^{\prime} \Vdash^{i n t} A$ then $w^{\prime} \Vdash^{i n t} B$

- $w \Vdash^{c l} \diamond A \Longleftrightarrow w R w^{\prime}$ and $w^{\prime} \Vdash^{c l} A$ for some $w^{\prime}$

- $w \Vdash^{i n t} \diamond A \Longleftrightarrow$ for all $w^{\prime}$, if $w \leq w^{\prime}$ then $w^{\prime} R v$ and $v \Vdash^{i n t} A$ for some $v$

The usual interpretations of negation are derived as follows.

- $w \Vdash^{c l} \neg A \Longleftrightarrow w \|^{c l} A$

- $w \Vdash^{\text {int }} \neg A \Longleftrightarrow$ for all $w^{\prime}$, if $w \leq w^{\prime}$ then $w^{\prime} \| f^{\text {int }} A$

Here are some remarks on the interpretation of $\diamond$.

Remark 3. 1. Intuitionistic interpretation of $\diamond$ requires to consider all $w^{\prime}$ above $x$. This comes from heredity condition usually assumed in intuitionistic logics.

2. This interpretation of $\diamond$ is the same as the one previously considered to reject distributivity of $\diamond$ over disjunction $(\diamond(A \vee B) \rightarrow \diamond A \vee \diamond B)$. This fromula is a theorem in classical modal logic, but sometimes it is considered intuitionistically unreasonable $[19,3]$.

3. Intuitionistic interpretation of $\diamond$ derives the duality of the two modality $\diamond p \equiv \neg \square \neg p$.

Definition 4. We write $\mathcal{F} \Vdash^{c l} A$ and say that $A$ is classically valid in $\mathcal{F}$ (or $\mathcal{F}$ classically validates $A$ ) if for all $V$ and $w$ it holds that $\mathcal{F}, V, w \Vdash^{c l} A$. Intuitionistic version of validity $\mathcal{F} \Vdash^{i n t} A$ is defined in the same way. 


\subsection{Algebraic Representation}

As usual, we can extend a valuation to the set of all formulas. Since there are two versions of semantics, we have two extensions of a valuation. We denote these extensions by $V^{c l}$ and $V^{i n t}$. The precise definition is:

$$
V^{*}(A)=\left\{w \mid \mathcal{F}, V, w \Vdash^{*} A\right\} .
$$

Below we introduce auxiliary notations and list some properties of $V^{c l}$ and $V^{\text {int }}$ for later use.

Definition 5. 1. For each $X \subseteq W$, we write $k(X)$ for the greatest upwardclosed subset of $X$. In other words, $k(X)=\{w \mid \leq[w] \subseteq X\}$.

2. For a valuation $V$, we define $V_{b}=k \circ V$. This is the greatest intuitionistic valuation less than or equal to $V$ (with respect to the pointwise set-inclusion).

3. For each $X \subseteq W$, we define $l_{R}(X)=\{w \mid R[w] \subseteq X\}$. Also, we use - for the set-theoretic complement, and $m_{R}(X)$ as a shorthand for $-l_{R}(-X)$.

It is easy to see that the following two hold for each valuation $V:(1) V_{b}$ is intuitionistic, and (2) $V_{b}=V$ if $V$ is intuitionistic. So by abuse of notation we just write $V^{\text {int }}$ to denote $\left(V_{b}\right)^{\text {int }}$ for non-intuitionistic valuation $V$.

It is easy to see that the following equalities hold for each valuation $V$.

$$
\begin{aligned}
V^{*}(A \wedge B) & =V^{*}(A) \cap V^{*}(B) & V^{*}(A \vee B) & =V^{*}(A) \cup V^{*}(B) \\
V^{*}(\square A) & =l_{R}\left(V^{*}(A)\right) & V^{c l}(A \rightarrow B) & =\left(-V^{c l}(A)\right) \cup V^{c l}(B) \\
V^{c l}(\diamond A) & =m_{R}\left(V^{c l}(A)\right) & V^{i n t}(p) & =V_{b}(p) \\
V^{i n t}(A \rightarrow B) & =k\left(\left(-V^{\text {int }}(A)\right) \cup V^{\text {int }}(B)\right) & V^{\text {int }}(\diamond A) & =k\left(m_{R}\left(V^{i n t}(A)\right)\right)
\end{aligned}
$$

Additionally, we would like to mention the two facts which play an important role in Section 4. First, a formula $A$ is valid in $\mathcal{F}$ if and only if $V^{*}(A)=W$ for all valuations $V$ on $\mathcal{F}$. This is clear from the definition. Second, the equality $l_{R} \circ k=l_{R}$ holds. This follows from the condition $(\leq ; R ; \leq)=R$ assumed in the definition of IM-frames.

\section{Axioms and Correspondents}

Having defined the semantics for intuitionistic modal logic, we are going to observe how well-known modal axioms can be characterized in terms of Kripke semantics. For some of them, their intuitionistic correspondents coincide with the classical ones, but for others they do not. After this observation, we discuss how this coincidence can be expressed formally, and introduce the notions of robustness. 


\begin{tabular}{ll|l} 
axiom & classical correspondent \\
\hline $\mathbf{5}$ & $\diamond p \rightarrow \square \diamond p$ & Euclidean (if $x R y$ and $x R z$, then $y R z$ ) \\
$\mathbf{B}$ & $p \rightarrow \square \diamond p$ & symmetric (if $x$, then $y R x$ ) \\
$\mathbf{T}_{\diamond}$ & $p \rightarrow \diamond p$ & reflexive $(x R x)$ \\
$\mathbf{4}_{\diamond}$ & $\diamond \diamond p \rightarrow \diamond p$ & transitive (if $x R y$ and $y R z$, then $x R z$ ) \\
$\mathbf{C D}$ & $\diamond p \rightarrow \square p$ & unique (if $x R y$ and $x R z$, then $y=z$ ) \\
$\mathbf{C}$ & $\diamond \square p \rightarrow \square \diamond p$ & confluent (if $x R y$ and $x R z$, then $\exists u .(y R u$ and $z R u$ ) $)$
\end{tabular}

Table 1: Examples of axioms whose classical and intuitionistic correspondent are not the same.

\subsection{Some Modal Axioms in IM-frames}

Some of the well-known axioms intuitionistically correspond to exactly the same property as the classical one. Typical examples of such axioms are $\mathbf{T}$ and $\mathbf{4}$, as mentioned in the introduction. For these two axioms, the classical method of deriving correspondents works perfectly in the intuitionistic case; take any possible world $w$ and consider $V(p)=R(w)$. This attempt works because there exists such an intuitionistic valuation $V$.

Also, $\mathbf{D}(\square p \rightarrow \diamond p)$ and $\mathbf{C 4}(\square \square p \rightarrow \square p$ ) have the same property, that is, their correspondents in the classical setting also apply to the intuitionistic case. D corresponds to seriality and $\mathbf{C 4}$ corresponds to density. These correspondences can be verified in similar ways to the cases of $\mathbf{T}$ and $\mathbf{4}$.

However, not all axioms necessarily correspond to the same property as the classical one. This can be observed in the work by Plotkin and Stirling in 1986 [14], although it is not mentioned explicitly. They considered a birelational Kripke semantics (different from ours), and gave a correspondent of LemmonScott axiom schema $\diamond^{k} \square^{l} p \rightarrow \square^{m} \diamond^{n} p$. According to their result, to express the correspondent of this schema we need to use both $R$ and $\leq$. Therefore we can see that (most of) the instances of Lemmon-Scott schema intuitionistically correspond to different properties from the classical case.

Although their result does not directly apply to our setting (because we are considering a different semantics from theirs), the situation is similar. Indeed, it is not difficult to find an axiom whose classical and intuitionistic correspondents are different. Some of them are listed in Table 1. All of these are instances of Lemmon-Scott axiom schema. In Figure 1 we list examples of IM-frames which intuitionistically validate these axioms but classically do not (the specified properties in Table 1 do not hold at $a$ of each frame). In this figure, solid and dotted arrows represent accessibility relations $R$ and $\leq$, respectively.

\subsection{Defining Robustness}

The series of examples above motivates the following question: which axiom corresponds to the same property in both classical and intuitionistic modal logics? This question will be discussed in the next section. In this subsection we are going to make a preparatory discussion on how to formalize the "sameness 


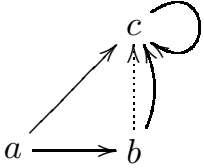

IM-frame for $\mathbf{5}$ which is not Euclidean

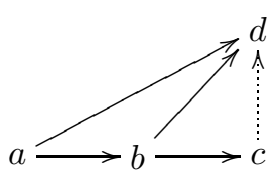

IM-frame for $\mathbf{4}_{\diamond}$ which is not transitive

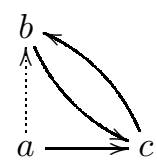

IM-frame for $\mathbf{B}$ which is not symmetric

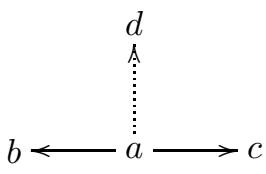

IM-frame for CD in which successor is not unique

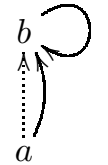

IM-frame for $\mathbf{T}_{\diamond}$ with irreflexive point

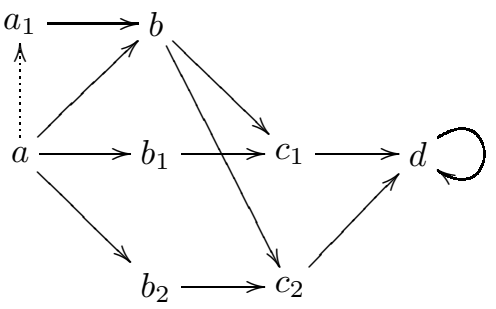

IM-frame for $\mathbf{C}$ without confluence

Figure 1: Examples of IM-frames which intuitionistically validate axioms, but not classically

of the classical and intuitionistic correspondents."

Let $A$ be an axiom, and assume that $A$ classically corresponds to a property $\varphi$, and intuitionistically corresponds to $\psi{ }^{3}$ If we write $\mathcal{F} \models \chi$ to mean that $\mathcal{F}$ satisfies a property $\chi$, this assumption means that

$$
\mathcal{F} \Vdash^{c l} A \Longleftrightarrow \mathcal{F} \models \varphi \text {, and } \mathcal{F} \Vdash^{i n t} A \Longleftrightarrow \mathcal{F} \models \psi
$$

for any IM-frame $\mathcal{F}$. In this setting, what does it mean for frame conditions $\varphi$ and $\psi$ to be the same? Here $\varphi$ and $\psi$ are properties of IM-frames, so it is natural to regard them as the same if and only if they are satisfied by exactly the same IM-frames. That is, $\varphi$ and $\psi$ are "the same" if and only if

$$
\mathcal{F} \models \varphi \Longleftrightarrow \mathcal{F} \models \psi
$$

holds for any IM-frame $\mathcal{F}$.

From the three equivalences above, we can see that the classical and intuitionistic correspondents for $A$ are the same if and only if

$$
\mathcal{F} \Vdash^{c l} A \Longleftrightarrow \mathcal{F} \Vdash^{i n t} A
$$

is the case for any $\mathcal{F}$.

Now we have arrived at the following definition.

\footnotetext{
${ }^{3}$ Formally, $\varphi$ and $\psi$ can be written as (second-order) sentences in an appropriate signature. The argument below can be written more formally by using this representation, but here we do not need the details.
} 
Definition 6. Let $A$ be a formula.

1. $A$ is said to be $C I$-stable if, for any IM-frame $\mathcal{F}$, if $\mathcal{F} \Vdash^{c l} A$ then $\mathcal{F} \Vdash^{\text {int }} A$.

2. $A$ is said to be $I C$-stable if, for any IM-frame $\mathcal{F}$, if $\mathcal{F} \Vdash^{\text {int }} A$ then $\mathcal{F} \Vdash^{c l} A$.

3. A is said to be robust if it is both CI-stable and IC-stable.

The intended meanings of these notions are explained as follows. Suppose that a formula $A$ classically corresponds to a certain property $\varphi$. Then, CIstability of $A$ means that if an IM-frame $\mathcal{F}$ satisfies $\varphi$, then $A$ is intuitionistically valid in $\mathcal{F}$. On the other hand, IC-stability of $A$ means that if an IM-frame $\mathcal{F}$ does not satisfy $\varphi$, then $A$ is not intuitionistically valid in $\mathcal{F}$. Accordingly, robustness of $A$ means that $A$ has the same correspondent $\varphi$ in classical and intuitionistic modal logics, as discussed above.

Example 7. 1. Axioms $\mathbf{T}, \mathbf{4}, \mathbf{D}$, and $\mathbf{C 4}$ are all robust.

2. $\mathbf{5}, \mathbf{B}, \mathbf{T}_{\diamond}, \mathbf{4}_{\diamond}, \mathbf{C D}$, and $\mathbf{C}$ are not robust. These axioms are all CI-stable, but not IC-stable.

Robustness of axioms in 1 follows from Theorem 12, and CI-stability in 2 follows from Proposition 14, clause 1.

Incidentally, the robustness is also characterized in terms of correspondents as follows.

Proposition 8. A formula $A$ is robust if and only if its intuitionistic correspondent for $A$ can be expressed without using $\leq$.

Proof. If $A$ is robust, then its intuitionistic correspondent is the same as the classical one, so "if" part is clear. We prove the other direction. For an IMframe $\mathcal{F}=\langle W, \leq, R\rangle$, define $\mathcal{F}^{*}:=\langle W, \Delta, R\rangle$, where $\Delta=\{(x, x) \mid x \in W\}$ is the identity relation. Since $\Delta$ is an ordering and $(\Delta ; R ; \Delta)=R$, this defines another IM-frame. It is easy to see that

1. if a property $\varphi$ can be expressed without $\leq$, then $\mathcal{F} \models \varphi \Longleftrightarrow \mathcal{F}^{*} \models \varphi$, and

2. $\mathcal{F} \Vdash^{c l} A \Longleftrightarrow \mathcal{F}^{*} \Vdash^{i n t} A$.

The assertion follows from these two facts.

\section{A Sufficient Condition for Robustness}

In this section, we give a sufficient condition for robustness. First we describe the main idea by considering a simple case, and next we extend this result to a more general form. There is an analogy between our result and a classical result by Sahlqvist [15], and the proof presented below is partly based on the algebraic proof of Sahlqvist's theorem [16, 5]. 


\subsection{Basic Idea}

Sahlqvist showed that an implication $A \rightarrow B$ corresponds to a first-order property, if $B$ is positive and $A$ satisfies a certain condition. This condition mentions positions of occurrences of $\rightarrow$ and $\square$; it restricts places in which these two connectives can occur.

Our result on robustness states, similar to Sahlqvist's theorem, an implication $A \rightarrow B$ is robust if $A$ and $B$ are positive and satisfies some conditions. These conditions are, again similar to Sahlqvist's one, phrased as conditions on occurrences of $\rightarrow, \square$, and $\diamond$.

Here we consider axioms of the form $A \rightarrow B$ only. For an axiom of this form, we have $\mathcal{F} \Vdash^{*} A \rightarrow B$ if and only if

$$
\forall V, w \cdot\left(\mathcal{F}, V, w \Vdash^{*} A \Longrightarrow \mathcal{F}, V, w \Vdash^{*} B\right)
$$

holds. Using the notation $V^{*}$, we can identify the validity of implication with the set inclusion, as shown in the following lemma.

Lemma 9. Let $A$ and $B$ be formulas. Then $\mathcal{F} \Vdash^{*} A \rightarrow B$ if and only if $V^{*}(A) \subseteq$ $V^{*}(B)$ for any valuation $V$ on $\mathcal{F}$.

So the robustness of $A \rightarrow B$ is equivalent to: $V^{c l}(A) \subseteq V^{c l}(B)$ for all $V$, if and only if $V^{\text {int }}(A) \subseteq V^{\text {int }}(B)$ for all $V$. This representation of robustness is convenient for our purpose, and it is the reason why we introduced algebraic representation.

To sketch the main idea, here we consider the simplest case. If we have $V^{c l}(A)=V^{\text {int }}(A)$ and $V^{c l}(B)=V^{\text {int }}(B)$ for every $V$, then $A \rightarrow B$ is clearly robust. When do these equalities hold? If we attempt to prove $V^{c l}(A)=$ $V^{\text {int }}(A)$ by induction on $A$, obviously there are problems in three cases: $p, \diamond$, and $\rightarrow$. In these cases $V^{c l}$ and $V^{\text {int }}$ do not agree, because $k$ is applied in clauses for $V^{\text {int }}$.

Here is a key observation: $l_{R}$ absorbs $k$, that is, $l_{R} \circ k=l_{R}$ holds, as mentioned at the end of Section 2. So, although in general we do not have

$$
V^{c l}(A)=V^{i n t}(A) \Longrightarrow V^{c l}(\diamond A)=V^{i n t}(\diamond A),
$$

instead we can say that

$$
V^{c l}(A)=V^{i n t}(A) \Longrightarrow V^{c l}(\square \diamond A)=V^{i n t}(\square \diamond A)
$$

holds, and similarly for $p$ and $\rightarrow$. This fact motivates the following definition.

Definition 10. An occurrence of an atom or a connective in a formula is said to be protected if it is immediately preceded by $\square$.

For example, in $\square p \wedge \square \diamond q$, the occurrences of $p$ and $\diamond$ are protected, but $q$ is not.

From the argument above, we can conclude that if all occurrences of atoms, $\diamond$, and $\rightarrow$ in $A$ and $B$ are protected, then $A \rightarrow B$ is robust. 4 is an example of such an axiom. In the next subsection, we are going to discuss how far this condition can be relaxed. 


\subsection{A Class of Robust Axioms}

Definition 11. A formula $A$ is said to be

1. positive if $A$ does not contain $\rightarrow$;

2. $\diamond$-protected if all occurrencees of $\diamond$ in $A$ are protected;

3. atom-protected if all occurrences of atoms, $\perp$ and $T$ excepted, in $A$ are protected;

4. protected if $A$ is both $\diamond$-protected and atom-protected.

Using these terminologies, we can state the main theorem as follows.

Theorem 12. Let $A$ be a protected positive formula, and $B$ a $\diamond$-protected positive formula. Then, $A \rightarrow B$ and $A \rightarrow \diamond B$ are robust.

Below we are going to prove this theorem.

Lemma 13. Let $A$ be a formula and $V$ a valuation.

1. If $A$ is positive, then $V^{\text {int }}(A) \subseteq V_{b}{ }^{c l}(A) \subseteq V^{c l}(A){ }^{4}$

2. If $A$ is positive and $\diamond$-protected, then $V^{\text {int }}(A)=V_{b}{ }^{c l}(A)$ and $V^{\text {int }}(\diamond A)=$ $k\left(V_{b}{ }^{c l}(\diamond A)\right)$.

3. If $A$ is atom-protected, then $V_{b}{ }^{c l}(A)=V^{c l}(A)$.

Proof. By induction on the construction of $A$, using the following facts:

1. positive formulas are constructed from atoms by $\wedge, \vee, \square$, and $\diamond$;

2. positive $\diamond$-protected formulas are constructed from atoms by $\wedge, \vee, \square$, and $\square \diamond ;$

3. atom-protected formulas are constructed from protected atoms, $\top$ and $\perp$ by freely applying any connectives.

In induction steps for 2 , we use the equality $l_{R} \circ k=l_{R}$.

Proposition 14. Let $A$ and $B$ be positive formulas.

1. If $B$ is $\diamond$-protected, then $A \rightarrow B$ and $A \rightarrow \diamond B$ are CI-stable.

2. If $A$ is protected, then $A \rightarrow B$ is IC-stable.

\footnotetext{
${ }^{4} V_{b}{ }^{c l}$ stands for $\left(V_{b}\right)^{c l}, \operatorname{not}\left(V^{c l}\right)_{b}$.
} 
Proof. Check that

$$
V^{i n t}(A) \subseteq V_{b}{ }^{c l}(A) \subseteq V_{b}{ }^{c l}(B)=V^{i n t}(B)
$$

and

$$
V^{i n t}(A) \subseteq k\left(V_{b}{ }^{c l}(A)\right) \subseteq k\left(V_{b}{ }^{c l}(\diamond B)\right)=V^{i n t}(\diamond B)
$$

for 1 , and

$$
V^{c l}(A)=V^{i n t}(A) \subseteq V^{i n t}(B) \subseteq V^{c l}(B)
$$

for 2, using Lemma 9.

From this proposition, it is clear that Theorem 12 holds. In the same way as Sahlqvist's theorem, we can extend this theorem.

Proposition 15. 1. If $A$ and $B$ are robust, then so is $A \wedge B$.

2. If $A$ and $B$ are robust and they do not share any atom, then $A \vee B$ is also robust.

3. If $A$ is robust, then so is $\square A$.

Proof. 1 and 2 are easy. For 3, take any IM-frame $\mathcal{F}$ and let $\mathcal{F}^{\prime}$ be the subframe of $\mathcal{F}$ generated by all $R$-successors (that is, all $w \in W$ such that for some $w^{\prime} \in W$ it holds that $w^{\prime} R w$ ). Then we can prove that $\mathcal{F} \Vdash^{*} \square A$ if and only if $\mathcal{F}^{\prime} \Vdash^{*} A$, from which robustness of $\square A$ follows.

Remark 16. Above we assumed that $A$ and $B$ are positive. Actually, in some ways this assumption can be relaxed a little. For example, if all occurrences of atoms, $\diamond$, and $\rightarrow$ in $A$ are protected, then the same result holds for nonpositive $A$, because for such $A$ it holds that $V^{c l}(A)=V^{\text {int }}(A)$, as discussed in the previous subsection. There would be some similar ways to extend our result.

However, this looks ad-hoc and makes things complicated, so here we do not investigate them further.

\section{Axioms from Access Control Logics}

In this section, we are going to consider some of the modal axioms appearing in security, and compare their strength using their correspondents. Although similar results have already been mentioned in recent literature $[2,6]$, the approach presented here is more systematic. This would be an example showing that a general theory on correspondence can be a useful foundation for comparing various modal axioms. 


\subsection{Modality and Access Control}

In the context of access control, modal logic has been considered as a basic framework to express access control policies, which determine whether a principal (user, program, machine, or other entity) may access a resource. Logics which express a policy as a formula is called access control logic, and have been studied recently.

In access control logics, a special operator, called says operator, plays a central role in expressing policies. This operator is used in the form $A$ says $s$, where $A$ is a principal and $s$ is a formula, and the whole expression is again a formula. Intuitively this formula means that " $A$ supports the statement $s$ " (although there seems to be some variations in exact interpretation of the says operator). Regarding $A$ says as a modality indexed by $A$, we can formalize an access control logic as a kind of multimodal logic.

As a logic for representing policies, access control logics usually require additional axioms concerning says operator other than necessitation and normality. For example, one may want to assume (admin says $s$ ) $\rightarrow s$ as an additional axiom on says operator. When $s$ represents an operation of deleting or modifying files, this axiom allows administrators to delete or modify files when they request.

Since different access control logics may interpret the says operator differently, there are several options in axiomatization of access control logics. To investigate room for choice, Abadi studied the consequence relation between some axioms in classical and intuitionistic modal logics [2]. His result implies that some intuitively natural principles derive an undesirable axiom (in particular, in classical setting) which make the logic degenerate. He also proved that some axioms do not prove such an axiom in intuitionistic setting, using proof-theoretic technique. What we are going to do is to obtain similar results more systematically, using results we have seen in the previous section.

\subsection{Axioms and their Robustness}

In what follows, we will write $\square_{A}$ instead of $A$ says, and suppress the principal annotation $A$ when it is not significant. We consider $\mathbf{C 4}$ having appeared before and the following axioms previously considered in relation to access control $\operatorname{logics}[2,10,6]$.

- Unit: $p \rightarrow \square p$

- Bind: $(p \rightarrow \square q) \rightarrow(\square p \rightarrow \square q)$

- Hand-off: $\square_{A}(B \Rightarrow A) \rightarrow(B \Rightarrow A)$

- Escalation: $\square p \rightarrow(p \vee \square \perp)$

It is easy to see that, under the presence of Unit, axioms Bind and C4 are (intuitionistically) equivalent. These three axioms come from lax logic, which is a version of intuitionistic modal logic [9]. Lax logic is known to be a 
logical foundation of computational lambda calculus $[12,11,4]$, and recently a calculus for access control, called CDD [1], has been proposed as a variant of computational lambda calculus.

In Hand-off axiom, a new connective $\Rightarrow$ appears. The formula $B \Rightarrow A$ means that " $B$ speaks for $A$," and $\Rightarrow$ is called "speaks for" operator. If we allow universal quantification over formulas, $\Rightarrow$ is defined by

$$
B \Rightarrow A \equiv \forall p \cdot\left(\left(\square_{B} p\right) \rightarrow\left(\square_{A} p\right)\right) .
$$

Hand-off axiom states that if a principal $A$ says that some principal $B$ speaks for $A$, then $B$ actually speaks for $A$. This axiom formalizes the delegation of authority.

Escalation is considered as an undesirable axiom, since a modality satisfying this axiom is considered degenerate; if we read $\square$ as "says," Escalation on $\square_{A}$ implies that either everything a principal $A$ says is true, or $A$ says anything [2].

Below we are going to compare the strength of these axioms, based on semantic method using robustness result. In particular, we consider which axiom does not imply which.

Before doing that, we adjust the definition of Kripke semantics so that it can interpret $\square_{A}$ and $\Rightarrow$. To interpret $\square_{A}$, instead of a single accessibility relation $R$, we employ a family of relations $R_{A}$ indexed by principals. Each $R_{A}$ is assumed to satisfy the same constraint as $R$ of IM-frames. Then, $\square_{A}$ is naturally interpreted, as the necessity with respect to $R_{A}$ in the new setting. We regard $B \Rightarrow A$ as a propositional constant, and thus it is interpreted as a fixed set of possible worlds. We define its classical interpretation by

$$
\llbracket B \Rightarrow A \rrbracket:=\left\{x \mid R_{A}[x] \subseteq R_{B}[x]\right\} .
$$

In other words, $B \Rightarrow A$ is true at $x$ if and only if $x R_{B} y$ implies $x R_{A} y$ for all $y$. This is consistent with the "definition" $B \Rightarrow A \equiv \forall p .\left(\left(\square_{B} p\right) \rightarrow\left(\square_{A} p\right)\right)$. Intuitionistic interpretation is just $k(\llbracket B \Rightarrow A \rrbracket)$.

Proposition 17. Escalation, C4 and Hand-off are robust, whereas Unit and Bind are not.

Proof. The robustness of these three axioms follows from Theorem 12 (note that $B \Rightarrow A$ can be treated as an atom).

Non-robustness of Unit and Bind are easily seen from the next proposition, in which we will see that the classical and intuitionistic correspondents for these axioms are distinct.

\subsection{Comparing Strength of Axioms}

It is not difficult to see the following correspondence in classical and intuitionistic modal logics.

Proposition 18. 1. C4 corresponds to: $R \subseteq R^{2}$. 
2. Unit classically corresponds to $R \subseteq \Delta$, where $\Delta$ is a diagonal relation, and intuitionistically corresponds to $R \subseteq \leq$.

3. Classically Bind is equivalent to Escalation, and therefore they have the same correspondence in classical setting. Intuitionistically Bind corresponds to:

$$
R[x] \subset R[\leq[x] \cap R[x]] .
$$

4. Escalation corresponds to: $R[x] \neq \emptyset \Longrightarrow x R x$.

5. Hand-off corresponds to the following condition:

$$
\left(\forall y \in R_{A}[x] .\left(R_{A}[y] \subseteq R_{B}[y]\right)\right) \Longrightarrow R_{A}[x] \subseteq R_{B}[x] .
$$

Since Escalation, C4, and Hand-off are robust, we do not distinguish classical and intuitionistic setting when considering their their correspondents. Similar results in a slightly different setting are also mentioned by Boella, Gabbay, Genovese and Torre [6].

From these results, the non-derivability of some axioms from other axioms in intuitionistic modal logic follows. For example:

- C4 does not imply Bind, and Bind does not imply Escalation.

- Hand-off does not imply $\mathbf{C 4}$ nor Unit. This is easily checked from the fact that a frame with $R_{A}=R_{B}$ for any principals $A$ and $B$ admits Hand-off, but not necessarily $\mathbf{C} 4$, nor Unit.

- Escalation does not imply Unit. This is also an easy consequence of the correspondence result. If we consider the following frame, it satisfies Escalation but not Unit, since $R[a]=\{a, b\} \nsubseteq\{a\}$.

$$
\text { Ca }
$$

- Unit does not imply C4 nor Hand-off. To see this, consider the following frame.

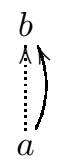

This satisfies Unit, but not C4. For Hand-off, regard the solid arrow as $R_{A}$ and let $R_{B}=\emptyset$. Then this frame satisfies Unit, but it falsifies Hand-off.

\section{Some More Examples}

In this section, we are going to discuss some more examples of modal axioms. 


\subsection{Limitation of Our Method}

The class of formulas covered by Theorem 12 and Proposition 15 contains all robust formulas listed in Section 3. However, there exists a robust formula outside the scope of the theorem, even if we consider axioms of the form $A \rightarrow B$ for positive $A, B$ only. For example, let $A=\square p$ and $B=p \vee \diamond p$, and consider the axiom $\mathbf{D}^{\prime}=A \rightarrow B$. Theorem 12 does not apply to $\mathbf{D}^{\prime}$ since $p$ on the righthand side of $\rightarrow$ is not protected. However, this axiom is robust, because $\mathbf{D}^{\prime}$ is valid in $\langle W, \leq, R\rangle$ if and only if $R$ is serial (in both classical and intuitionistic interpretations).

For this axiom, our method used above does not seem to work. This is because, if $A \rightarrow B$ can be proved to be robust in our method, it actually means a little more than robustness; for such $A$ and $B$, it holds that $V_{b}{ }^{c l}(A) \subseteq$ $V_{b}{ }^{c l}(B) \Longleftrightarrow V^{i n t}(A) \subseteq V^{i n t}(B)$. However, for the choice of $A$ and $B$ above, this equivalence does not hold. Indeed, take the frame

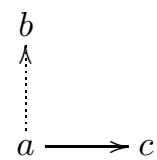

and consider $V(p)=\{b, c\}$. Then, after a little calculation we obtain $V^{c l}(A)=$ $V^{\text {int }}(A)=V^{c l}(B)=\{a, b, c\}$ but $V^{\text {int }}(B)=\{b, c\}$.

$\mathbf{D}^{\prime}$ is not an axiom motivated from some meaningful application. It is constructed as a counterexpmale, and does not seem a natural axiom since $p$ on the right-hand side is redundant. There could be more meaningful counterexpmale, but at the time of writing we do not have such an example.

\subsection{IC-Stable, but not CI-stable Axioms}

The examples of non-robust axioms that appeared above are all CI-stable, but not IC-stable. It is a natural question whether there exists an axiom which is IC-stable, but not CI-stable.

Perhaps the simplest example is $\square p \rightarrow \diamond \diamond p$. It is easy to check that this axiom is classically valid in a frame $\langle W, R\rangle$ if and only if $R \cap R^{2}$ is serial (in other words, $R$ has the property that for all $w \in W$ there exists $v \in W$ such that both $w R v$ and $w R^{2} v$ holds). On the other hand, there exists an IM-frame in which $R \cap R^{2}$ is serial, but the said axiom is not valid. For example, consider the frame

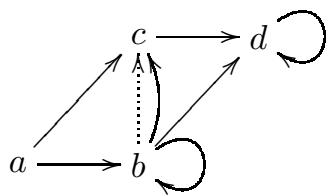

and a valuation $V(p)=\{b, c\}$. Then we have $a \Vdash^{i n t} \square p$, but $a \| \nvdash^{i n t} \diamond \diamond p$. To check the latter, note that $c$ has no successor satisfying $p$. So $c \forall^{i n t} \diamond p$, and from heredity we also have $b \mid \forall^{i n t} \diamond p$. This means that $a$ has no successor satisfying $\diamond p$, so $a$ does not satisfy $\diamond \diamond p$. 


\subsection{Löb axiom}

Above we considered axioms of the form $A \rightarrow B$ where $A$ and $B$ are positive. As an example of robust formulas which does not have this form, we consider Löb axiom (GL), which takes the form $\square(\square p \rightarrow p) \rightarrow \square p$. In classical modal logic, this axiom is known to correspond to the conjunction of transitivity and non-existence of infinite path [5]. Let us take a look at the sketch of the proof.

For transitivity, assume $u R v R w$ and let $V$ be a valuation such that $x \in V(p)$ if and only if neither $x=v$ nor $x=w$ holds. Then, from the contrapositive of GL there exists some $R$-successor of $u$ at which $\square p \rightarrow p$ does not hold. On the other hand we can show that, at any world other than $w$ we have $\square p \rightarrow p$. Therefore $u R w$ needs to hold.

For non-existence of infinite path, define $V$ by: $w \in V(p)$ if and only if there is no infinite path starting from $w$. Then under this valuation $\square p \rightarrow p$ is true everywhere, so applying GL we obtain $V(p)=W$. This means that there is no infinite path.

This proof is done in the classical setting, but almost the same method works for IM-frame, too. We only need to fix the definition of $V$ in the first part. Instead of "neither $x=v$ nor $x=w$ " we need to consider "neither $x \leq v$ nor $x \leq w$ " because otherwise $V$ may fail to be an intuitionistic valuation.

Interestingly, we can also use algebraic argument to prove the same result. A little calculation shows that GL is classically valid if and only if all valuations $V$ satisfy

$$
l_{R}\left(\left(-l_{R}(V(p))\right) \cup V(p)\right) \subseteq l_{R}(V(p)),
$$

and intuitionistically

$$
l_{R}\left(\left(-l_{R}(V(p))\right) \cup k(V(p))\right) \subseteq l_{R}(V(p)) .
$$

Therefore, to see GL is robust it is sufficient to show that

$$
k(X \cup Y)=k(X \cup k(Y)),
$$

where $X=-l_{R}(V(p))$ and $Y=V(p)$. The right-to-left inclusion is obvious. For the converse, take $x \in k(X \cup Y)$, and let $U$ be the set of all upper bounds of $x$. Then we have $U \subseteq X \cup Y$, hence $U \backslash X \subseteq Y$. Actually we can say $U \backslash X \subseteq k(Y)$, because the left-hand side is the intersection of the two upwardclosed sets, namely $U$ and $-X=l_{R}(V(p))$. Therefore we have

$$
U=(U \cap X) \cup(U \backslash X) \subseteq X \cup k(Y),
$$

and hence $x \in k(X \cup k(Y))$.

\subsection{Grzegorczyk axiom}

As another famous example of an axiom of a compilcated form, we have Grzegorczyk axiom (Grz) $\square(\square(p \rightarrow \square p) \rightarrow p) \rightarrow p$. Classically, Grz is known 
to correspond to reflexive and transitive frames in which there is no nontrivial infinite path [5] (by "nontrivial" infinite path we mean a path $x_{1} R x_{2} R$.. such that $x_{n} \neq x_{n+1}$ for any $\left.n\right)$. This condition resembles to that for $\mathbf{G L}$, but unlike GL, Grz is not robust.

It holds that if an IM-frame $\mathcal{F}$ is reflexive, transitive, and has no nontrivial infinite $R$-path, then $\mathcal{F}$ validates Grz. However, the converse does not hold; there exists an IM-frame with nontrivial infinite $R$-path, in which $\mathbf{G r z}$ is valid. For example, let $W$ be the set of all natural numbers and both $\leq$ and $R$ be the usual ordering on natural numbers. Then we can easily check that, from heredity, $p$ and $\square p$ are equivalent at each world in this frame. So in this peculiar frame Grz is valid, although it contains nontrivial infinite path $(1,2,3, \cdots$, for example).

Actually, in IM-frame, Grz corresponds to the conjunction of reflexivity, transitivity and the following condition: there exists no pair of an upwardclosed (with respect to $\leq$ ) set $X$ and an infinite path $x_{1} R x_{2} R \cdots$, such that $x_{n} \in X$ if and only if $n$ is even. By transitivity, this fact means that Grz is valid if and only if there is no infinite path on which truth of $p$ can alter infinitely many times.

\section{Concluding Remarks}

\section{$7.1 \quad$ Summary}

We studied correspondence of axiom and properties of frames in intuitionistic modal logic, and there are two main observations. First, the sameness of classical and intuitionistic correspondents can be captured by the equivalence in classical and intuitionistic semantics. Second, there exists a syntactically defined class of axioms, for whose members the correspondents in the classical and intuitionistic settings are the same.

The semantics considered in this work is the one defined by Wolter and Zakharyaschev [21]. It seems that the condition $(\leq ; R ; \leq)=R$ plays an important role. In particular, we used the equality $l_{R} \circ k=l_{R}$ in the proof of Lemma 13, which is a consequence of this condition.

To give a class of robust axioms syntactically, the main strategy we took is to restrict occurrences of "problematic" constructs (atoms, $\rightarrow$ and $\diamond$ ), which requires intuitionistic counterpart $\leq$ to interpret. A similar method is used in Sahlqvist's theorem, in which mainly $\rightarrow$ and $\square$ are restricted.

At first sight the class we gave may seem to be small because it strongly restricts occurrences of an atom and $\diamond$. However, it contains most part of standard robust axioms. At the time of writing, we could find only a few examples of robust formulas outside this class. To make significant improvement in the current result, some new idea would be required.

Also, as a possible application, we have considered modal axioms from security. Using model theoretic argument together with robustness results, we have compared strength of these axioms. This demonstrates that the result presented 
in this paper can be a tool for investigating modal logic in the intuitionistic setting.

\subsection{Similar Result for Other Semantics}

Our argument above is based on IM-frame, and depends on the choice of semantics. Here we briefly discuss how to develop a similar result for other semantics.

Besides the clause in Definition 2, there is another standard interpretation of $\square A$ :

$$
w \Vdash^{i n t} \square A \Longleftrightarrow \text { if } w \leq w^{\prime} \text { and } w^{\prime} R w^{\prime \prime} \text {, then } w^{\prime \prime} \Vdash^{i n t} A \text {. }
$$

In this case, we do not need any constraint on $\leq$ and $R$ (like $(\leq ; R ; \leq)=R$ assumed in IM-frames). This version is also found in the literature $[8,17,19$, 3 , 14]. If we define $w \Vdash^{i n t} \square A$ in this way, we need to use $\leq$ as well as $R$ to express correspondents concerning $\square$ (the situation is the same as the problem we found in Section 4 , where occurrences of $\diamond$ caused a problem). As a result, it seems that most part of the classical correspondence results are not true for this semantics; even $\mathbf{T}$ and $\mathbf{4}$ are excluded. So, if the notion of robustness is left unchanged, the same problem for this semantics would not be interesting.

One possible approach in this setting is to use the result on IM-frames indirectly, by giving a translation from non-IM-frames to IM-frames. If we do not consider $\diamond$, or we admit duality $\diamond p \equiv \neg \square \neg p$ (which is a theorem in IM-frame semantics), it is not difficult to translate the semantics defined on non-IM-frame into IM-frame semantics. This is because, from heredity, we have $w \Vdash^{i n t} \square A$ in this semantics if and only if "if $w(\leq ; R ; \leq) w^{\prime}$, then $w^{\prime} \Vdash^{\text {int }} A$ " holds. Therefore what we actually need to focus on is not $R$, but the composite $R^{\prime}=(\leq ; R ; \leq)$. Regarding $R^{\prime}$ as new $R$, we obtain IM-frame semantics. Therefore, if an axiom $X$ corresponds to some property $\varphi(R)$ in IM-frame semantics, then the same axiom corresponds in non-IM-frame semantics to $\varphi(\leq ; R ; \leq)$. So we can say that if $X$ is robust, then its intuitionistic correspondent in non-IM-frames can be obtained by replacing all occurrences of $R$ in the classical correspondent with $(\leq ; R ; \leq)$.

\subsection{Local Version of Robustness}

In Section 3 we have defined robustness of an axiom $A$ by

$$
\forall \mathcal{F} .\left(\mathcal{F} \Vdash^{c l} A \Longleftrightarrow \mathcal{F} \Vdash^{i n t} A\right) .
$$

This is a global notion in the sense that it concerns global validity only. We could also consider its local version like

$$
\forall \mathcal{F}, w \cdot\left(w \Vdash^{c l} A \Longleftrightarrow w \Vdash^{i n t} A\right)
$$

( $w$ ranges over the set of possible worlds of $\mathcal{F}$ ), which seems to be a natural definition of local robustness. However, axioms satisfying this condition would 
be rare. This is because the intuitionistic interpretation has heredity, while the classical one does not. For example, consider an IM-frame consisting of two comparable points $a \leq b$, and assume $a$ is reflexive and $b$ is not $(R=\{(a, a),(a, b)\})$. Then, classically $\mathbf{T}, \mathbf{4}$ and $\mathbf{D}$ are locally valid at $a$, but intuitionistically they are not.

One possibility to adjust the definition of local robustness is to consider the following condition:

$$
\forall \mathcal{F}, w \cdot\left(\left(\forall w^{\prime} \cdot w \leq w^{\prime} \Longrightarrow w^{\prime} \Vdash^{c l} A\right) \Longleftrightarrow w \Vdash^{i n t} A\right)
$$

If we define local robustness of $A$ by this condition, then the same result as Theorem 12 is proved by a similar argument.

\subsection{Related Work}

It seems that the problem considered in this paper has not been studied before. However, there are a few studies concerning correspondence for concrete modal axioms in intuitionistic settings.

One of such studies has been done by Sotirov [18]. He introduced two modal accessibilities $R$ and $R^{*}$ independently to interpret $\square$ and $\diamond$, and gave corresponding properties for various axioms in terms of three accessibility relations $\leq, R$ and $R^{*}$. These axioms include $\mathbf{T}, \mathbf{T}_{\diamond}, \mathbf{4}, \mathbf{4}_{\diamond}, \mathbf{B}$, and $\mathbf{D}$.

In his result, because of the existence of independent $R^{*}$, occurrences of $\diamond$ do not introduce extra $\leq$ in expressing the corresponding properties. As a result, in contrast with ours, it would be the case that the occurrences of $\diamond$ does not interfere robustness (but an appropriate definition of robustness in his setting is not obvious because of the independence of $R$ and $R^{*}$ ).

He also considered a lot of axioms containing nested $\rightarrow$ (and $\neg$ ), which we did not handle. In his setting, as well as ours, nested implications cause nonrobustness of axioms (that is, we need to mention $\leq$ to express the correspondents). For example, $\square A \rightarrow \neg \neg A$, which classically corresponds to reflexivity, corresponds to the following property: if $x \leq y$, then there exists $z$ such that $y \leq z$ and $x R z$.

The work by Plotkin and Stirling [14] mentioned in Section 3 is another example. They defined a birelational Kripke semantics, discussed some concrete axioms, and identified their correspondents.

According to their result, the Lemmon-Scott axiom schema $\diamond^{k} \square^{l} p \rightarrow \square^{m} \diamond^{n} p$ corresponds to the following property:

if $w R^{k} u$ and $w R^{m} v$, then there exists $u^{\prime}$ and $x$ such that $u \leq u^{\prime}, u^{\prime} R^{l} x$, and $v R^{n} x$.

From this result we can see that, in their setting, as well as ours, many axioms would correspond to properties different from the classical case.

They also considered the axiom $\diamond p \wedge \diamond q \rightarrow \diamond(p \wedge \diamond q) \vee \diamond(q \wedge \diamond p)$, which, when added to $\mathrm{S} 4$, results in $\mathrm{S} 4.3$. The correspondent of this axiom they gave 
is also different from the classical one previously known (if $x R y$ and $x R z$, then either $y R z$ or $z R y$ holds).

Also, it would be worth noting that they pointed out that it is unlikely that classically equivalent axioms ( $\square p \rightarrow p$ and $p \rightarrow \diamond p$, for example) always correspond to the same property. Their result shows that this is indeed the case.

\section{References}

[1] Martín Abadi. Access control in a core calculus of dependency. Electronic Notes in Theoretical Computer Science, 172:5-31, 2007.

[2] Martín Abadi. Variations in access control logic. In Ron van der Meyden and Leendert van der Torre, editors, Deontic Logic in Computer Science, volume 5076 of Lecture Notes in Computer Science, pages 96-109. Springer Verlag, 2008.

[3] Natasha Alechina, Michael Mendler, Valeria de Paiva, and Eike Ritter. Categorical and Kripke semantics for constructive S4 modal logic. In Laurent Fribourg, editor, Computer science logic : 15th International Workshop, CSL 2001, 10th Annual Conference of the EACSL, Paris, France, September 10-13, 2001 : proceedings, volume 2142 of Lecture Notes in Computer Science, pages 292-307. Springer Verlag, 2001.

[4] P. N. Benton, Gavin Bierman, and Valeria de Paiva. Computational types from a logical perspective. Journal of Functional Programming, 8(2):177193, 1998.

[5] Patrick Blackburn, Maarten de Rijke, and Yde Venema. Modal Logic. Cambridge University Press, 2002.

[6] Guido Boella, Dov M. Gabbay, Valerio Genovese, and Leetndert van der Torre. Fibred security language. Studia Logica, 92(3):395-436, August 2009.

[7] Milan Božić and Kosta Došen. Models for normal intuitionistic modal logics. Studia Logica, 43(3):217-245, September 1984.

[8] W. B. Ewald. Intuitionistic tense and modal logic. Journal of Symbolic Logic, 51(1):166-179, 1986.

[9] Matt Fairtlough and Michael Mendler. Propositional lax logic. Information and Computation, 137(1):1-33, 1997.

[10] Deepak Garg and Martín Abadi. A modal deconstruction of access control logics. In Roberto Amadio, editor, Foundations of Software Science and Computational Structures, volume 4962 of Lecture Notes in Computer Science, pages 216-230. Springer Verlag, 2008. 
[11] Satoshi Kobayashi. Monad as modality. Theoretical Computer Science, 175:29-74, 1997.

[12] Eugenio Moggi. Computational lambda calculus and monads. In Proceedings of IEEE Symposium on Logic In Computer Science (LICS'89), pages 14-23, Washington, DC, 1989. IEEE Computer Society Press.

[13] Hiroakira Ono. On some intuitionistic modal logics. Publications of the Research Institute for Mathematical Sciences, 13(3):687-722, 1977.

[14] Gordon Plotkin and Colin Stirling. A framework for intuitionistic modal logics. In Joseph Y. Halpern, editor, Proceedings of the 1986 conference on Theoretical aspects of reasoning about knowledge, pages 399-406. Morgan Kaufmann Publishers Inc., 1986.

[15] Henrik Sahlqvist. Correspondence and completeness in the first and second order semantics for modal logic. In Stig Kanger, editor, Proceedings of the Third Scandinavian Logic Symposium, pages 110-143, North-Holland, Amsterdam, 1975.

[16] G. Sambin and V. Vaccaro. A new proof of Sahlqvist's theorem on modal definability and completeness. The Journal of Symbolic Logic, 54(3):992999, September 1989.

[17] Alex K. Simpson. The Proof Theory and Semantics of Intuitionistic Modal Logic. PhD thesis, University of Edinburgh, 1994.

[18] Vladimir H. Sotirov. Modal theories with intuitionistic logic. In Mathematical Logic, Proceedings of the Conference on Mathematical Logic, Dedicated to the Memory of A. A. Markov (1903-1979), Sofia, September 22-23, 1980, pages 139-171, Sofia, 1984.

[19] Duminda Wijesekera. Constructive modal logics I. Annals of Pure and Applied Logic, 50:271-301, 1990.

[20] Frank Wolter and Michael Zakharyaschev. On the relation between intuitionistic and classical modal logics. Algebra and Logic, 36:73-92, 1997.

[21] Frank Wolter and Michael Zakharyaschev. Intuitionistic modal logics as fragments of classical bimodal logics. Logics at work, Essays in honour of Helena Rasiowa, pages 168-186, 1999. 